\title{
El concepto de unidad fraseológica
}

\author{
M. ${ }^{a}$ Auxiliadora Castillo Carballo \\ Universidad de Sevilla
}

\section{INTRODUCCIÓN}

El proceso de codificación consiste en la selección de palabras que, unidas, tienen la finalidad de expresar aquellas ideas que el hablante quiere o tiene necesidad de transmitir. El arsenal léxico del que dispone, unas veces, es usado para construir combinaciones libres en las que prima la ausencia de criterios de restricción en la elección de los elementos que conforman el acto de habla, con la salvedad de las leyes semánticas y gramaticales que rigen las lenguas; $\mathrm{y}$, otras, combinaciones fijas en las que funcionan mecanismos de reproducción de grupos léxicos con fijación variable, pero restringidos por el sentido o por su construcción.

En el discurso se conjugan los dos procedimientos, el de creación y el de reproducción, que en la investigación lingüística se corresponden con pares conceptuales como técnica del discurso frente a discurso repetido para Eugenio Coseriu (1981, pp. 297-302), lenguaje literal y lenguaje no literal para Fernando Lázaro Carreter (1980, pp. 149-171) o con the open choice principle y the idiom principle de John Sinclair (1991, pp. 109-115).

A lo largo de los años, se ha intentado realizar estudios, unas veces más acertados que otros, que dieran luz al intrincado universo que ocupa la fraseología1. Delimitar sus fronteras, clarificar los tipos que la inte-

1 Una perspectiva histórica de los estudios fraseológicos permite discernir entre dos grandes esferas: la comprendida por la interrelación de las investigaciones soviéticas y europeas, por un lado, y, por otro, por las aportaciones de la lingüística norteamericana. A la primera pertenecen autores como A. Schmid (1989, pp. 121-127), L. Zgusta (1967, pp. 578-587 y 1971), P. Kühn (1984, pp. 175-235), R. Gláser (1986, pp. 41-52), O. Alexandrova y S. Ter-Minasova (1987), D. O. Dobrovol'skij (1988), entre otros; y a la segunda, C. F. Hockett (1958), J. J. Katz y P. M. Postal (1963, pp. 275-283), Wallace L. Chafe (1968, pp. 109-127), Bruce Fraser (1970, pp. 22-42), A. Makkai (1972), Chitra Fernando y Roger Flavel (1981), y otros. 
gran, y dar definiciones precisas que carecieran de cualquier ambigüedad han sido objetivos que muchas veces no se han podido cumplir. Tal vez sea el reciente auge de esta disciplina lo que explique que su investigación, hasta hace muy pocos años, se encontrara en los albores; lo que ha dado lugar a una gran variedad terminológica, referida tanto al vocablo general ${ }^{2}$ que debe abarcar estos hechos lingüísticos como a las distintas denominaciones de cada uno de los fenómenos individuales. Incluso, esto ha trascendido a la considerable diversidad de clasificaciones desde diversos puntos de vista. Sin embargo, desde el principio ha existido un consenso para nombrar a la disciplina, constituida como tal en los años cuarenta por los lingüistas soviéticos, que engloba el estudio de las combinaciones de palabras con el término fraseología.

\section{LA UNIDAD FRASEOLÓGICA Y EL DICCIONARIO}

El constante descuido en los estudios lexicológicos de los aspectos fraseológicos ha influido inevitablemente en la práctica lexicográfica. Así pues, los diccionarios han acogido, entre sus artículos, las combinaciones de palabras con cierta fijación de modo bastante irregular, recurriendo, en muchos casos, a etiquetados pocos exhaustivos y vacilantes.

Julio Fernández-Sevilla señaló, por su parte, la necesidad de elaborar diccionarios que dieran cabida a unidades más amplias que la palabra $^{3}$. Igualmente, aseguró que estas no deben ser inventariadas en reper-

2 En la filología española se barajan nombres como los de unidad fraseológica, expresión pluriverbal, unidad pluriverbal lexicalizada y habitualizada, unidad léxica pluriverbal, expresión fija o fraseologismo. No creo que sea de extrema importancia la elección de uno frente a los demás, pues, aunque es cierto que algunos de ellos parecen ser más apropiados que otros -bien porque han sido utilizados, a su vez, como subtipos dentro del conjunto, o porque su denominación tan solo señala una característica de tales combinaciones-, lo verdaderamente relevante es que su utilización tenga presente y claros los límites de esta parcela lexicológica denominada fraseología. No obstante, prefiero la denominación de unidad pluriverbal, seguida de la de unidad fraseológica, aunque esta tiene últimamente una mayor difusión, sobre todo en Europa, debido a los fraseólogos soviéticos.

${ }^{3}$ Para referirse a estas unidades utiliza el término lexía, que reconoce haber tomado de Pottier, pero subraya que con el sentido de "secuencia estable y fija de unidades léxicas y/o gramaticales, funcionalmente equivalente a una palabra que la lengua no posee o el habla no actualiza" equivalente a lo que tradicionalmente se ha llamado locución. Vid. Julio Femández-Sevilla (1974), p. 21. 
torios independientes, ni incluidas al final del artículo de diccionario, pues si se parte de la base de que la lexía es funcionalmente una palabra, esta debe tener el mismo estatus que el resto de las entradas, con igual independencia, y ocupando el lugar que le corresponda de acuerdo con la naturaleza del repertorio en el que se incluya. Además, puntualizó que no “importará que la ortografía normativa preceptúe representar gráficamente estas unidades de manera discontinua, con espacios blancos entre los elementos componentes" (Fernández-Sevilla, 1974, p. 22).

Sin embargo, ante estas clarividentes palabras, resulta incomprensible que se plantee que esta determinación ocasionará al lexicógrafo algunos problemas, pensando que no siempre le será fácil saber cuál es el lugar que la lexía debe ocupar en los diccionarios, independientemente de que el criterio de ordenación sea el alfabético o el ideológico.

Creo que esta objeción no se sustenta en bases sólidas si se tiene presente lo dicho anteriormente, es decir, que estas secuencias de unidades léxicas más o menos fijas y estables deben ser ordenadas según la configuración formal que impere en cada una de las obras lexicográficas. De todos modos, sus palabras tal vez se refieran a la gran heterogeneidad que subyace en estas unidades, lo que generaría ciertas dudas a la hora de elegir qué elemento del conjunto debería constituir la palabra-clave que sirviese de referencia inequívoca para facilitar al usuario la búsqueda; todo ello sin obviar las posibles variantes que de una misma combinación fija pudieran existir, así como la habitual rivalidad entre varias unidades léxicas de una misma secuencia en cuanto a su carga semántica, que imposibilitaría la fácil elección de una frente a las otras como palabra ordenatriz ${ }^{4}$. A todo esto hay que sumarle uno de los escollos fundamentales de la fraseología: la sistematización de tipos sustentada sobre parámetros definitorios claros y precisos.

Por tanto, resulta necesario ahondar en cuáles se podrían considerar características fundamentales y afines a los distintos fenómenos fraseológicos.

${ }^{4}$ Igualmente, hay que tener en cuenta que tanto en los estudios sobre fraseología como en los diccionarios se descuida habitualmente la correcta distinción del llamado contorno lexicográfico respecto de los elementos que constituyen la unidad fraseológica, tendiendo a integrarlo como uno más de sus elementos, o bien a prescindir de él. Incluso, a veces, ante la presencia de varios, se llega a cometer verdaderas incoherencias enmarcando entre corchetes tan solo uno. Esto sucede especialmente con aquellas unidades en las que se reproduce con un pronombre enclítico un objeto indirecto (*darle a alguien mala espina algo / dar [a alguien] mala espina [algo]). Véase J. Martínez Marín (1991), pp. 123-124 y (1996), pp. 60-61. 


\section{CARACTERÍSTICAS DE LAS UNIDADES FRASEOLÓGICAS}

Con frecuencia, se suele señalar que una secuencia de palabras será considerada unidad fraseológica en la medida en que cumpla, al menos, dos condiciones esenciales: fijación e idiomaticidad. Sin embargo, se pueden aducir otros rasgos que están presentes en estas combinaciones léxicas, y que se irán enumerando a continuación.

\subsection{FIJACIÓN}

La fijación referida a estas combinaciones de palabras ha de ser entendida en el sentido de algo ya hecho que el hablante almacena y tiende a reproducir sin descomponerlo en sus elementos constituyentes. Es decir, se trata de "la propiedad que tienen ciertas expresiones de ser reproducidas en el hablar como combinaciones previamente hechas - tal como las estructuras prefabricadas, en arquitectura" (Zuluaga, 1975, p. 230).

Zuluaga distingue diversos tipos de fijación, y señala que los más habituales en español son la inalterabilidad del orden de los componentes (de armas tomar / *de tomar armas, a ciencia cierta / * a cierta ciencia); la invariabilidad de alguna categoría gramatical, relacionada con el tiempo verbal, la persona, el número, el género (quien mucho abarca poco aprieta / *quien mucho abarca poco apretó, arrojar la toalla / *arrojar las toallas); la inmodificabilidad del inventario de los elementos integrantes, tanto por supresión como por adición (a ojo de buen cubero / *a ojo de cubero, dar una de cal y otra de arena / *dar una de cal por las mañanas y otra de arena por las tardes); y su insustituibilidad (de cabo a rabo / * de extremo a rabo, de rompe y rasga /*de destroza y rasga); así como la imposibilidad de transformación (duro de pelar / *duro de peladura, enajenación mental / *enajenación de la mentalidad) ${ }^{5}$. Pero esta tipificación, al hilo de la planteada por Harald Thun (1978), tan solo se refiere a la fijación interna material, un subgrupo dentro de la que llama fijación interna, que comparte el mismo estatus con la interna de contenido. El otro tipo sería la fijación externa, en la que aprecia varios modelos, la situacional, la analítica, la pasemática y la posicional6.

5 Alberto Zuluaga, (1975), p. 227. Vid. también del mismo autor (1980), pp. 97-98.

${ }^{6}$ Cf. Gloria Corpas Pastor (1997), pp. 23-24, y véanse los ejemplos. 
Lingüistas como Wallace L. Chafe (1968), F. J. Newmeyer (1974, pp. 326-342) o Maurice Gross (1982, pp. 151-185), han recurrido para explicar la fijación a la defectividad sintáctica, entendida como el rechazo de determinadas operaciones lingüísticas que sí funcionan en el campo de las combinaciones libres de palabras y que, para algunos, se sustenta en el sentido global de sus componentes (es el caso de a pie juntillas, a la pata la llana, a ojos vista, etc.). Esta concepción también es compartida por Mel'čuk, quien, además, propone que dicha fijación se puede medir teniendo en cuenta el grado de probabilidad con el que alguno de los componentes de una expresión puede predecir la presencia de los otros elementos. Y asegura que la fijación de una secuencia de palabras será total si su elemento indicador no funciona fuera de la expresión, no obstante, no cree que se pueda dar una oposición extrema entre lo que es fijo y lo que no lo es, sino que existe una gradación que da sentido a la existencia de estadios intermedios. Pero, en definitiva, piensa que las investigaciones estadísticas son las que pueden establecer límites fiables? ${ }^{7}$

\subsection{LEXICALIZACIÓN}

Por otra parte, se ha señalado la estrecha relación entre la fijación y la especialización semántica o lexicalización, ocasionada esta por adición o por supresión de significado, una vez que se ha establecido una identificación entre una unidad pluriverbal y su valor semántico en un entorno determinado. A este respecto, señala Corpas Pastor que una combinación de palabras se fija primero y después es cuando se convierte en una unidad potencial susceptible de modificar su significado. Por ello, la especialización semántica implica fijación, pero esta no presupone a la otra8. Así pues, en una expresión como encontrar o hallar [uno] la horma de su zapato, se ha producido previamente una consolidación del uso de las palabras que la componen hasta constituirse un significado unitario que no puede ser analizado por la suma de sus elementos integrantes. De este modo, si consultamos el Diccionario académico, veremos el proceso de especialización semántica que se ha producido:

1. fr. fig. y fam. Encontrar lo que le acomoda o lo que desea.

${ }^{7}$ Cf. Alberto Zuluaga (1980), p. 66.

8 Vid. Gloria Corpas Pastor (1997), p. 26. 
2. fig. y fam. Tropezar con alguien o con algo que se le resista o que se oponga a sus mañas o artificios 9 .

\subsection{IDIOMATICIDAD}

La idiomaticidad ha sido entendida de dos maneras diferentes. Por un lado, responde, en el sentido etimológico, a lo que es propio y peculiar de una lengua y, por otro, se puede interpretar como el rasgo semántico característico de ciertas construcciones fijas, en las que su significado no puede ser deducido a partir de los elementos que la forman, y así es como debe entenderse en el ámbito fraseológico. Sentencia Zuluaga que "la expresión idiomática es un signo complejo pero no, simultáneamente y desde el punto de vista funcional, un complejo de signos. Los componentes de ésta no se comportan en ella como signos lingüísticos, propiamente, sino, más bien, como componentes formales de un signo" (1980, pp. 123-124).

Por otro lado, la idiomaticidad de una unidad pluriverbal no está directamente relacionada con la cantidad de elementos idiomáticos que posea, pues si solo uno funciona idiomáticamente la unidad será idiomática. En este sentido, se considera que una palabra es idiomática cuando, por pertenecer a etapas sincrónicas previas a la actual de una lengua determinada, tiene valor únicamente dentro de la unidad fraseológica correspondiente, careciendo, por tanto, de vida léxica fuera de ella. Igualmente, se consideran idiomáticos los préstamos léxicos, así como las deformaciones fónicas, morfológicas, apócopes, pues en el seno de una expresión determinan su sentido idiomático ${ }^{10}$. Reflejo de ello son expresiones como mondo y lirondo, a la chita callando, sin ton ni son, por fas o por nefás, etc.

Pese a que la idiomaticidad se ha tratado habitualmente como uno de los rasgos más esenciales de las unidades fraseológicas, hay que tener presente que no todas ellas son idiomáticas, pues, más bien, "se trata de una característica potencial, no esencial, de este tipo de unidades" (Corpas Pastor, 1997, p. 27).

10 Cf. Mario García-Page Sánchez (1990), pp. 279-290. 


\subsection{OTROS RASGOS: DE LA FRECUENCIA DE COAPARICIÓN A LA PLURIVERBALIDAD}

Si fijación e idiomaticidad se han erigido a lo largo de la investigación fraseológica como las características fundamentales comunes a los hechos lingüísticos que abarca, lo cierto es que existen otras peculiaridades que tienen también una gran relevancia.

Así, tanto la frecuencia de coaparición como la frecuencia de uso son especialmente importantes, pues la frecuencia de algunas combinaciones es un factor determinante en la creación de unidades fraseológicas. Íntimamente relacionado con esto hay que tener presente la repetición, según la cual las unidades fraseológicas se convierten en secuencias de uso general en una comunidad de hablantes. Si bien, puntualiza Zuluaga, se debe hablar, más bien, de reproducción, pues las unidades fraseológicas son repetidas sin cambiar su forma, con el fin de poder distinguirla de las posibles repeticiones de un contenido sin ajustarse a una forma rígida (1980, p. 26). En este sentido, el diccionario, en la medida en que registra estas unidades, sirve de testimonio de dicha reproducción ${ }^{11}$.

También se ha hablado de la intraducibilidad de las unidades fraseológicas, pues su fijación puede estar en el origen de hechos históricos o situaciones concretas, lo que motiva que al hablante que aprende una lengua le resulte muy difícil comprender su sentido y, por tanto, memorizarlas y, mucho más, reproducirlas adecuadamente. Ello también viene condicionado por la opacidad de significado como consecuencia de la idiomaticidad, en la que el sentido de los elementos constituyentes se diluye en beneficio del que designa el conjunto.

Con la fijación fraseológica contrasta la variación léxica, pues parece contrariar "una de las 'leyes' fundamentales que gobiernan el código de las expresiones fijas: la inmodificabilidad o inalterabilidad de las mismas" (García-Page Sánchez, 1996, p. 477). Esto pone de manifiesto que la fijación en las unidades fraseológicas es relativa. Pues, a veces, las variantes son tan violentas que hacen dudar de si se trata de una unidad pluriverbal o no. En este sentido, señala García-Page que existen estructuras locucionales que, perteneciendo a la sintaxis libre, presentan menos variantes, y lo ejemplifica con la secuencia quedarse en casa, donde observa que el núcleo verbal no puede ser conmutado por sustantivos de similares rasgos semánticos: *quedarse en oficina, * quedarse en colegio.

11 O. Alexandrova y S. Ter-Minasova (1987). 
Por esta razón, cree que es el sentido idiomático o semiidiomático el que se convierte en índice del carácter fraseológico de una expresión pluriverbal (1996, pp. 477-478).

Con cierta asiduidad, se han considerado variantes fraseológicas aquellas modificaciones de una unidad pluriverbal que no violan su sentido y se insertan dentro de la norma. Por su parte, Carneado Moré ha señalado que estamos ante una variación fraseológica cuando se dan modificaciones que no alteran los rasgos esenciales de la unidad en cuestión, evitando que se pueda producir una identificación de la variante con una estructura diferente, como si se tratase de otro fraseologismo. Y puntualiza que "en ningún caso la modificación de un giro fraseológico lo altera si se realiza en aquellos puntos de la estructura que no cumplen una función diferenciadora" (1985, pp. 270-271).

De acuerdo con sus criterios observa que, entre otras, las variantes que se dan en las unidades fraseológicas son de tipo morfológico, es decir, cambios en su forma y no en su función; de tipo léxico, las más productivas por el amplio desarrollo en la lengua de las relaciones paradigmáticas; y las variantes por extensión, que se caracterizan por la adición u omisión de algunos de sus elementos.

No obstante, ante las posibles confusiones entre los cambios que se pueden experimentar en las unidades fraseológicas, se ha distinguido entre variantes en sentido amplio, o pseudovariantes, y variantes en sentido estricto ${ }^{12}$.

A las primeras corresponden las transformaciones que se manifiestan mediante un cambio de significado, tanto categorial como léxico, también denominadas modificaciones (tomar el pelo / tomadura de pelo, meter la pata / metedura de pata); igualmente, corresponden a las variantes en sentido amplio las llamadas series (de buena fe / de mala fe, al por mayor / al por menor); así como las unidades que, aunque compartan una equivalencia de significado, posean estructuración y elementos totalmente diferentes (tomar las de Villadiego / poner pies en polvorosa, poner de vuelta y media [a alguien] / dejar [a alguien] a la altura de una zapatilla); tampoco hay que olvidar las variantes diatópicas, diafásicas o diastráticas, entre otras.

En cuanto a las variantes en sentido estricto, su caracterización viene dada por presentarse dentro de una misma lengua funcional, sin cambios de significado, y con absoluta libertad e independencia de los

12 Vid. Alberto Zuluaga (1980), pp. 106-110 y (1975), pp. 237-243. 
contextos, por la sustitución parcial de alguno de sus componentes y por la fijación o restricción de los elementos potenciales disponibles en la operación de conmutabilidad. Este último rasgo corrobora que la existencia de variantes no implica un menor grado de fijación, pues las posibles sustituciones están también fijadas (por ejemplo, importar [algo] un bledo / un pito, dormir como un tronco / como un lirón, de punta a punta / cabo $\sim$ rabo / pe pa).

Por otro lado, algunas modificaciones de las unidades fraseológicas no deben ser entendidas como variantes, sino como una manifestación de su grado de fijación, "así, cuanto mayor es su fijación, y por ende su institucionalización, más posibilidades hay de que sufran una modificación en el discurso, y de que tal modificación y su efecto sean reconocidos por los hablantes" (Corpas Pastor, 1997, p. 29).

Y por último, otro rasgo que también concierne a las unidades tratadas es el concepto de pluriverbalidad, según el cual están constituidas por dos o más palabras ${ }^{13}$, lo que justifica en gran medida la denominación elegida, en algunos casos, para referirse a estos fenómenos lingüísticos.

\section{CONCLUSIÓN}

En definitiva, de todo lo expuesto se puede decir que la fraseología es una disciplina lingüística que tiene por objeto de estudio el conjunto de aquellas unidades léxicas formadas por más de dos vocablos con separación gráfica, y que se caracterizan por:

la alta frecuencia de uso, y de coaparición de sus elementos integrantes; por su institucionalización, entendida en términos de fijación y especialización semántica; por su idiomaticidad y variación potenciales; así como por el grado en el cual se dan todos estos aspectos en los distintos tipos (Corpas Pastor, 1997, p. 20).

Así mismo, no hay que olvidar, como se ha señalado más arriba, que una de las grandes dificultades con la que se encuentra la fraseología es con la delimitación exhaustiva de los diferentes tipos que albergan las genéricamente denominadas unidades fraseológicas. No obstante, en la actualidad se ha establecido una de las más serias tipologizaciones de

13 Cf. Julio Casares (1992); A. P. Cowie (1991), pp. 101-106; E. Gates, (1988), pp. 99-106; Humberto Hernández (1989); H. Jackson (1989). 
estas unidades ${ }^{14}$, atendiendo a la distinción tripartita entre norma, sistema y habla, según la cual habría que contar con tres grandes grupos o esferas que se corresponderían con las llamadas colocaciones o combinaciones de unidades léxicas fijadas solo en la norma; las locuciones o unidades del sistema; y los enunciados fraseológicos, que están fijados en el habla y que constituyen enunciados completos, a diferencia de los otros dos, que poseen un estatus equivalente al sintagma, razón por la que necesitan coaparecer con otros elementos para poder subsistir. Todos ellos estarían constituidos, además, por una serie de subtipos, que intentan aprehender todas las posibles manifestaciones de la combinatoria léxica.

En este sentido, una correcta aplicación de los parámetros teóricos que caracterizan a la globalidad de las unidades fraseológicas es lo que puede permitir que se establezca, aunque no fácilmente, una correcta tipologización. 


\section{BIBLIOGRAFÍA}

Alexandrova, O. y S. Ter-Minasova (1987): English Syntax (Collocation, Colligation and Discourse), Moscú, Universidad de Moscú.

CARneado Moré, Zoila (1985): "Notas sobre las variantes fraseológicas", Anuario L/L (La Habana), 16, pp. 269-277.

CASARES, Julio (1992): Introducción a la lexicografia moderna, Madrid, C.S.I.C., $3^{\mathrm{a}}$. ed., [1950].

Corpas PAstor, Gloria (1997): Manual de fraseología española, Madrid, Gredos.

CoSERIU, Eugenio (1981): Lecciones de lingüística general, Madrid, Gredos.

CowIE, A. P. (1991): "Multiword Units in Newspaper Language", Cahiers de l'Institut de Linguistique de Louvain, 17, 1-3, pp. 101106.

Chafe, Wallace L. (1968): "Idiomaticity as an anomaly in the Chomskyan paradigm", Foundations of Language, 4, pp. 109-127. Dobrovol'sKIJ, D. O. (1988): Phraseologie als Objekt der Universalienlinguistik, Linguistische Studien, Leipzig.

Fernández-Sevilla, Julio (1974): Problemas de lexicografía actual, Bogotá, Instituto Caro y Cuervo.

Fernando, Chitra y Roger Flavel (1981): On Idiom. Critical Views and Perspectives, Exeter, University of Exeter.

FRASER, Bruce (1970): "Idioms within a transformational grammar", Foundations of Language, 6, pp. 22-42.

GARCÍA-PAGE SÁNCHEZ, Mario (1990): "Léxico y sintaxis locucionales: algunas consideraciones sobre las palabras "idiomáticas", Estudios humanísticos. Filología, 12, pp. 279-290.

GARcíA-PAGE SÁNCHEZ, Mario (1996): "Sobre las variantes fraseológicas en español", Revista canadiense de estudios hispánicos, XX, 3, pp. 477-490.

GATES, E. (1988): "The treatment of multiword lexemes in some current dictionaries of English", en M. Snell-Hornby (ed.), ZüriLEX'86 Proceeding. Papers read at the EURALEX International Congress, Tubinga, Francke, pp. 99-106.

GLÂSER, R. (1986): “A plea for phraseo-stylistics”, en D. Kastovsky y A. Szwedek (eds.), Linguistics across Historical and Geographical Boundaries. In Honour of Jacek Fisiak on the Occasion of his 
Fiftieth Birthday, I, Linguistic Theory and Historical Linguistics, Trends in Linguitics. Studies and Monographs, 32, Berlín-Nueva York-Amsterdam, Mouton de Gruyter, pp. 41-52.

Gross, Maurice (1982): "Une classification des phrases figées du français", Revue québécoise de linguistique, 11, 2, pp. 151-185. HERNÁNDEZ, Humberto (1989): Los diccionarios de orientación escolar. Contribución al estudio de la lexicografia monolingüe española, Tubinga, Max Niemeyer.

HocketT, C. F. (1958): A Course in Modern Linguistics, Nueva York, The MacMillan Company.

JACKSON, H. (1989): Words and Their Meaning, Londres-Nueva York, Longman.

Katz, J. J. y P. M. Postal (1963): "Semantik interpretation of idioms and sentences contining them", M.I.T. Quaterly Progress Report, 70, pp. 275-283.

KÜHN, P. (1984): "Pragmatische und lexikographische Beschreibung phraseologischer Einheiten: Phraseologismen und Routinenformeln", en H. E. Wiegand (ed.), Studien zur neuhochdeutschen Lexicographie $I V$. Germanistische linguistik, 1-3, 83, Hildesheim, Georg Olms, pp. 175-235.

LÁZARo CARRETER, Fernando (1980): Estudios de lingüística, Barcelona, Crítica.

MAKKAI, A. (1972): Idiom Structure in English, La Haya, Mouton. NEWMEYER, F. J. (1974): “The Regularity of Idiom Behaviour”, Lingua, 34, 4, pp. 326-342.

Real Academia Española (1992, 1996): Diccionario de la lengua española, $21^{\mathrm{a}}$. ed. y CD-ROM ed., Madrid, Espasa Calpe.

SCHMID, A. (1989): "Wörterbücher als Hilfe zur Übersetzung von Phraseologismen", en M. Snell-Hornby y E. Pölh (eds.), Translation and Lexicography. Papers read at the EURALEX Colloquium held at Innsbruck 2-5 July 1987, John Benjamins, Paintbrush, Euralex, pp. 121-127.

Sinclair, John (1991): Corpus, Concordance, Collocation, OxfordNueva York, Oxford University Press.

THuN, Harald (1978): Probleme der Phraseologie. Untersuchungen zur wiederholten Rede mit Beispielen aus den Französischen, Italienischen, Spanischen und Romänischen, Beihefte zur Zeitschrift für Romanische Philologie 168, Tubinga, Max Niemeyer. 
Zgusta, Ladislav (1967): "Multiword Lexical Units", Word, 23, pp. 578587.

ZGUSTA, Ladislav (1971): Manual of lexicography, Janua Linguarum. Studia Memoriae Nicolai Van Wijk Dedicata Series Maior, $n^{\circ} 39$, La Haya, Mouton.

ZuluaGA, Alberto (1975): "La fijación fraseológica", Thesaurus, XXX, pp. 225-248.

ZuluaGa, Alberto (1980): Introducción al estudio de las expresiones fijas, Studia Románica et linguistica; 10, Frankfurt a. M., Berna, Cirencester/U. K.: Lang. 